\title{
First Ohmic Discharge Assisted with RF Power in QUEST Spherical Tokamak
}

\author{
Osamu MITARAI, Kazuo NAKAMURA ${ }^{1)}$, Saya TASHIMA ${ }^{1)}$, Makoto HASEGAWA ${ }^{1)}$, \\ Hiroshi IDEI $^{1)}$, Mizuki SAKAMOTO ${ }^{1)}$, Kazuaki HANADA ${ }^{1)}$, Hideki ZUSHI ${ }^{1)}$, Kohnosuke SATO ${ }^{1)}$, \\ Masaki ISHIGURO ${ }^{1)}$, Hai Qing LIU ${ }^{1)}$, Yuta HIGASHIZONO ${ }^{1)}$, Aki HIGASHIJIMA ${ }^{1)}$, \\ Hisatoshi NAKASHIMA $^{1)}$, Shoji KAWASAKI ${ }^{1)}$, Yuichi TAKASE ${ }^{2)}$, Takashi MAEKAWA ${ }^{3)}$ \\ and Nobuhiro NISHINO ${ }^{4}$ \\ Liberal Arts Education Center, Kumamoto Campus, Tokai University, 9-1-1 Toroku, Kumamoto 862-8652, Japan \\ ${ }^{1)}$ Research Institute for Applied Mechanics, Kyushu University, 6-1 Kasuga-koen, Kasuga 816-8580, Japan \\ ${ }^{2)}$ Graduate School of Frontier Sciences and Graduate School of Science, University of Tokyo, Kashiwa 277-85611, Japan \\ ${ }^{3)}$ Graduate School of Energy Science, Kyoto University, Kyoto 606-8502, Japan \\ 4) Department of Engineering, Hiroshima Univeristy, Higashi-Hiroshima 739-8527, Japan
}

(Received 23 October 2009 / Accepted 17 November 2010)

\begin{abstract}
Ohmic plasma currents of up to $17 \mathrm{kA}$ with a discharge duration of $0.32 \mathrm{~s}$ have been obtained in the Kyushu University Experiment with Steady-State Spherical Tokamak (QUEST) with the help of electron cyclotron wave (ECW) and cancellation coils (CCs). The CCs, originally installed to create a field null in the plasma breakdown phase, are essential for producing plasma current in QUEST. Although the ohmic coil current is initially biased and then reduced completely to zero to induce the plasma current in $15-20 \mathrm{~ms}$, we demonstrate that the flat top of the plasma current exceeding $20 \mathrm{~ms}$ is maintained by the vertical field after the ohmic current is switched off. This type of operation is quite favorable for extending pulsed operation to the steady state by electron Bernstein wave current drive (EBCD).
\end{abstract}

(c) 2011 The Japan Society of Plasma Science and Nuclear Fusion Research

Keywords: spherical tokamak, current start-up, ohmic clamp, cancellation coil, ECW, vertical field

DOI: $10.1585 /$ pfr.6.1402003

\section{Introduction}

Plasma current ramp-up has been recognized as a major difficulty in low-aspect ratio spherical tokamaks (STs) [1] because of insufficient room for a central solenoid (CS) on the inboard side of the torus. A new concept has been proposed for ramping up the plasma current using vertical field and heating power [2,3]; it was successfully demonstrated on the JT-60U tokamak [4-6] and the TST-2 [7] and MAST [8] STs. However, as steady state was not maintained in these experiments, an experimental demonstration of steady-state operation in an ST is necessary.

On the other hand, "ohmic clamp" experiments are basic experiments related to plasma current ramp-up by vertical field that is sometimes conducted in the initial phase of experiments of this type because of their relatively simple setup $[4,9]$. In these experiments, the ohmic coil current is kept constant and heating power is applied. Any plasma current ramp-up during the "ohmic clamp" phase may be due to the vertical field effect because the non-inductive drive method usually needs longer time to show its effect [4]. The first "ohmic clamp" experiments in an ST were performed in National Spherical Torus Ex- periment (NSTX) [10]. However, the plasma current was not sufficiently ramped up to demonstrate its effect. The reasons are discussed in a previous paper but are not yet clear [10]. On the other hand, in the newly operational QUEST machine, ohmic discharge assisted with RF power corresponds exactly with the "ohmic clamp" experiments described in this paper. Here, we demonstrate that the plasma current can be ramped up by the vertical field effect in the QUEST tokamak.

The QUEST tokamak has been designed to achieve steady-state operation with a plasma current $\geq 20 \mathrm{kA}$ by using electron Bernstein wave current drive (EBCD) and to study plasma-wall interactions in high-temperature $\left(\sim 500^{\circ} \mathrm{C}\right)$ environments [11]. Experimental operating scenarios can be categorized as (1) pure ohmic discharge, (2) ohmic and RF current drive, (3) pure RF start-up to the steady state, and (4) vertical-field-assisted plasma current start-up and RF current drive to the steady state.

To start up the plasma current in a tokamak, two methods are used to create a poloidal field (PF) null in the plasma region. The first is to use PF coils, such as the shaping vertical field coil (PF26 coil in QUEST) and divertor coils, to reduce the stray field from the ohmic transformer. However, as the initial current di- 
rection of PF26 coil produces an opposite vertical field to the equilibrium field, its current polarity should be quickly changed for equilibrium control, which requires a high-voltage bi-directional circuit. This method is used in NSTX, for example [12]. In several other operational or planned tokamaks worldwide, such as Alcator C-MOD [13], EAST [14], KSTAR [15], ITER [16], and JT-60SA [17], well-predicted preprogrammed control of many PF coil currents is or will be used to create a field null regime and a subsequent plasma shape, which are essential for this method.

The second method for creating a field null regime is to use additional cancellation coils (CCs) to reduce the stray field from the ohmic transformer. Because the ohmic transformer and the CCs are connected in series, the power supply circuit cost can be reduced and no ripple effect appears even if one is present in the power circuit. Therefore, the operation is simpler than the first method, even during the flat-top phase, because vertical field control does not depend on the ohmic transformer current. This second method has been used in ASDEX Upgrade [18], DIII-D [19], JT-60U [20], TRIAM-1M [21], TST-2 [22], and Globus-M [23], and will be used in the SST-1 [24] and KTM [25] tokamaks.

In this paper, we report the first ohmic discharge using an ohmic transformer and $\mathrm{CC}$ assisted with $\mathrm{RF}$ power. A flat top of a 17-kA plasma current and a $320 \mathrm{~ms}$ duration has been achieved in the first experimental campaign. Although the ohmic pulse duration is very short, a much longer discharge duration has been obtained because of the vertical field induction effect. In QUEST, the induction duration of the ohmic current is presently as short as $20 \mathrm{~ms}$ because of power supply circuit limitations. After $20 \mathrm{~ms}$, the ohmic current is switched off and becomes zero, which corresponds to the "ohmic clamp" experiments. This type of scenario with "ohmic clamp" discharge assisted with RF power is favorable for future steady-state operation with RF current drive.

\section{Experimental Layout}

In QUEST, the major radius is $R \sim 0.68 \mathrm{~m}$, the minor radius is $a \leq 0.40 \mathrm{~m}$, the elongation is $\kappa \leq 1.8$, and the toroidal field is $B_{\mathrm{t}} \leq 0.5 \mathrm{~T}$. A launcher for electron cyclotron waves (ECWs), designed for electron Bernstein wave $(\mathrm{EBW})$ heating at a frequency of $8.2 \mathrm{GHz}$, is installed on the outboard side of the torus in QUEST. The cut-off density for the O-mode is $0.83 \times 10^{18} \mathrm{~m}^{-3}$.

In QUEST, CCs are connected in series to the ohmic coil to reduce the poloidal stray field from the ohmic transformer during the breakdown phase, as shown in Fig. 1. As the power supply circuit is modified from that of the highaspect ratio tokamak TRIAM-1M, the current waveform of the PF26 vertical field coil is not fully controllable. Even if the waveform is controlled, a large current ripple in the PF26 vertical field coil power supply may not be able to

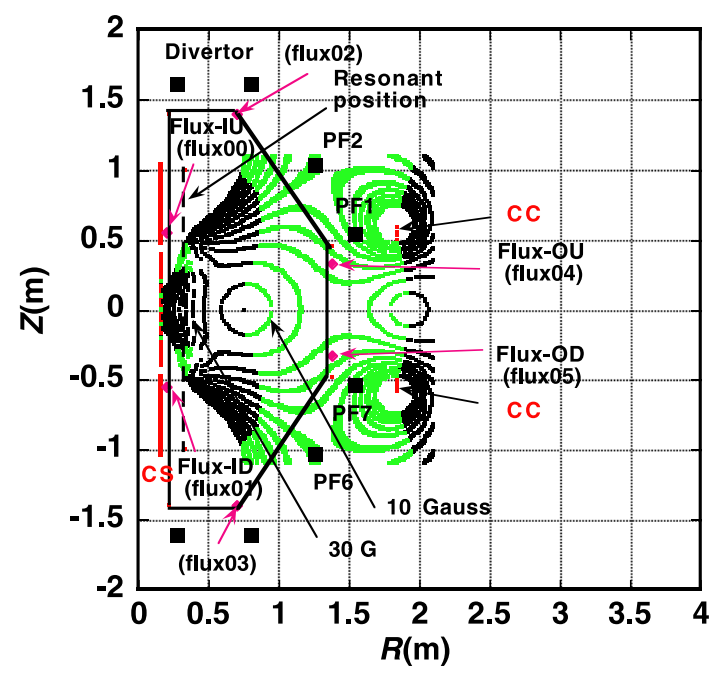

Fig. 1 Magnetic field strength and the location of poloidal field coils in QUEST for $I_{\mathrm{CS}}=8 \mathrm{kA}$ and $I_{\mathrm{PF} 26}=0 \mathrm{kA}$. Green regimes indicate the vertical field opposite to the equilibrium field.

reduce the stray field from the ohmic coil. An additional CC (with a maximum of four turns) can cancel the poloidal stray field from the ohmic coil, as shown in Fig. 1. However, the best position for the $\mathrm{CC}$ is already occupied by other equipment and only the space outside of the PF17 coils is available.

A CC with one turn was selected to create a small field null region in the central area of the vacuum chamber, as shown in Fig. 1. However, with a weak toroidal field of $B_{\mathrm{t}}=0.14 \mathrm{~T}$ at $R=0.68 \mathrm{~m}$ in the first phase of the experiments, the resonant position of $8.2 \mathrm{GHz} \mathrm{RF}$ was at $33 \mathrm{~cm}$. This position is in the inward regime and outside of the null field regime, but in the higher ohmic induction electric field regime. Plasma current start-up from the inward position has a smaller plasma inductance, which is suitable for ramping up the plasma current.

\section{Experimental Results}

\subsection{Initial discharges}

At the second shot after starting the ohmic discharge experiments, a short pulse of plasma current up to $\sim 3.5 \mathrm{kA}$ was produced (\#1448); this increased to $\sim 8.5 \mathrm{kA}$ after four shots (\#1452) and then suddenly a long discharge of $10 \mathrm{kA}$ and $\sim 250 \mathrm{~ms}$ (\#1456) was obtained, as shown in Fig. 2. The long discharges can be reproduced by adjusting the vertical magnetic field by using PF26 coils to optimize the plasma current. Plasma cross section images taken by a fast TV camera are shown in Fig. 3.

In the reproducible discharges, vertical field is applied during the breakdown phase. A typical applied vertical field is $74 \mathrm{G}\left(I_{\mathrm{PF} 26}=0.25 \mathrm{kA}\right.$ in Fig. $\left.5(\mathrm{c})\right)$ at $R=$ $0.68 \mathrm{~m}$. Therefore, as shown in the calculated magnetic field strength distribution in Fig.4, a wide null regime 


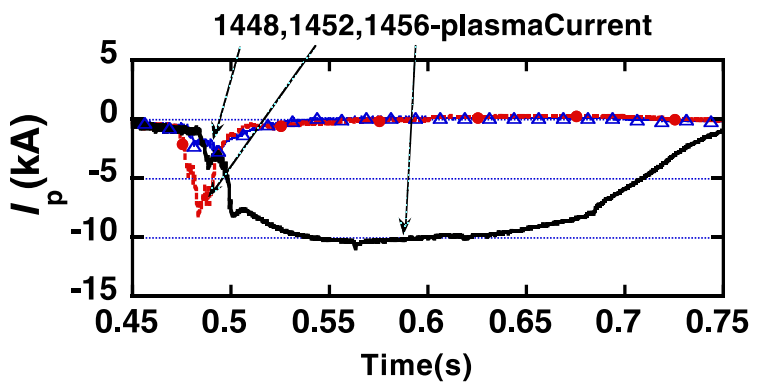

Fig. 2 Plasma current evolution at second (\#1448), fifth (\#1452), and tenth shots (\#1456) on the first day of Ohmic discharge experiments in QUEST. (a) $t=0.49771 \mathrm{~s}$

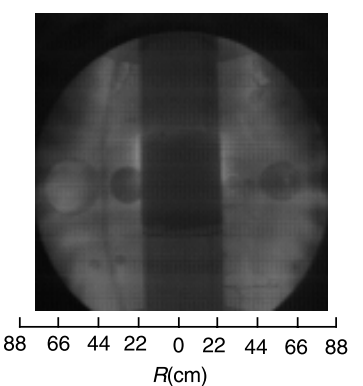

(c) $t=0.499185 \mathrm{~s}$

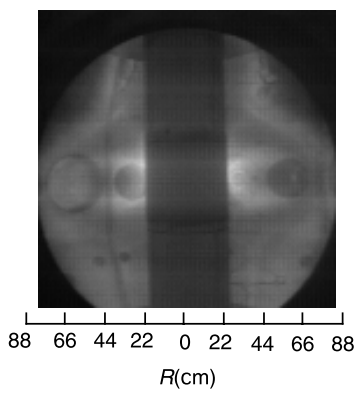

(b) $t=0.498815 \mathrm{~s}$

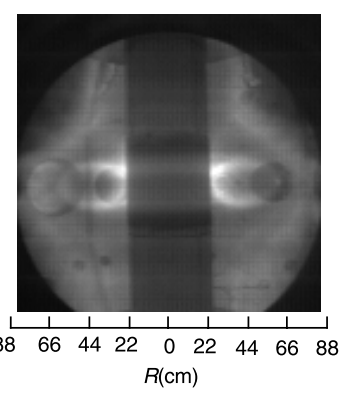

(d) $t=0.500222 \mathrm{~s}$

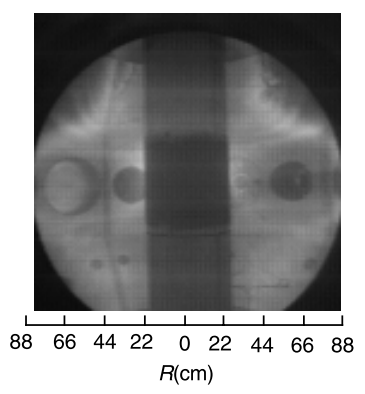

Fig. 3 Fast TV camera images of initial plasma current start-up phase of first long ohmic discharge in QUEST. (\#1456)

disappears when the vertical field is applied. Note that the vertical field induced by the vacuum chamber current is negligible at the breakdown phase because the vacuum chamber current crosses zero, as seen later in Figs. 6, 8, and 13. However, two small null points remain at $z \sim \pm 0.47 \mathrm{~m}$ and at the resonant line, as indicated by the dashed line in Fig. 4, although their role is unclear.

\subsection{Larger ohmic coil bias current of $I_{\mathrm{CS}}=$ $8 \mathbf{k A}$}

After careful adjustments of the vertical field, a plasma current of up to $15 \mathrm{kA}$ (\#1932) was obtained. The overall discharge waveforms of various parameters are shown in Fig. 5. As shown in Fig. 5 (h), RF power was applied from $0.4 \mathrm{~s}$ to $0.56 \mathrm{~s}$, and the ohmic coil current was increased to $8.3 \mathrm{kA}$ for biasing and ramped down to

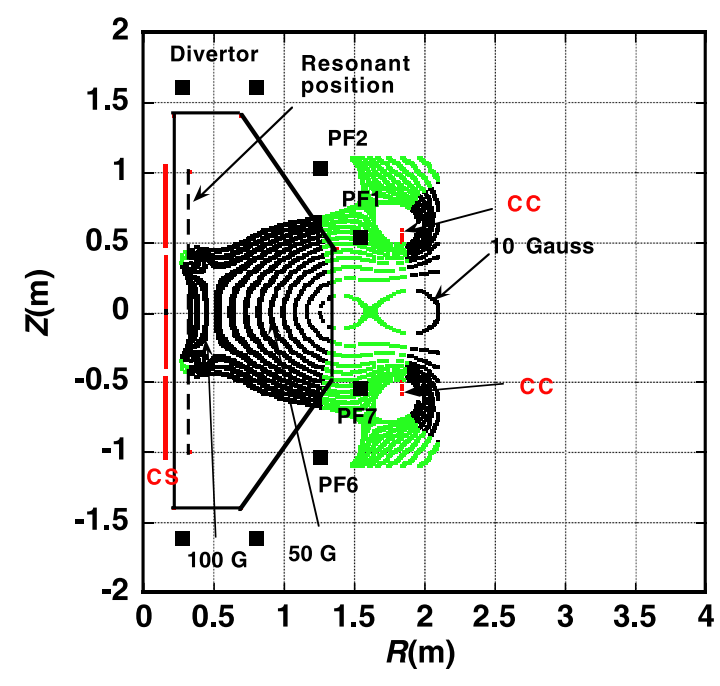

Fig. 4 Magnetic field strength for $I_{\mathrm{CS}}=8 \mathrm{kA}$ and $I_{\mathrm{PF} 26}=$ $0.25 \mathrm{kA}$. Green regimes indicate the vertical field opposite to the equilibrium field.

zero at $0.5 \mathrm{~s}$ for inducing the plasma current (Fig. 5 (b)), as performed in standard ohmic discharge operation in a tokamak. The plasma current starts at $t=0.48 \mathrm{~s}$ and increases to $\sim 10 \mathrm{kA}$ in $20 \mathrm{~ms}$ with two bumps; it then slowly increases to $15 \mathrm{kA}$ after the ohmic coil current becomes zero. A vertical field was applied at the breakdown phase; therefore, the null field regime is not created in an actual discharge, as shown in Fig. 4. For a PF26 coil current of $I_{\mathrm{PF} 26} \sim 0.3 \mathrm{kA}$, the estimated vertical field is $B_{\mathrm{v}} \sim 137 \mathrm{G}$ at the resonant position.

The flux measured on the ohmic transformer is $\sim 0.08$ Vs (see Fig. 5 (f)), which is close to the estimated value using the axial strength of the magnetic field from the $I_{\mathrm{CS}}$ current, as $\Phi=\pi r^{2} B_{\mathrm{z}}=0.073 \mathrm{Vs}$. The resistive flux is $\Phi_{\mathrm{R}}=C_{\mathrm{E}} \mu_{\mathrm{o}} R I_{\mathrm{p}} \sim 0.006 \mathrm{Vs}$ for an Ejima coefficient $C_{\mathrm{E}}=0.7, R=0.68 \mathrm{~m}$, and $I_{\mathrm{p}}=10 \mathrm{kA}$. Because the inductive flux is estimated to be $L_{\mathrm{p}} I_{\mathrm{p}}=0.6 \times 10^{-6} \times 10^{4} \sim$ $0.006 \mathrm{Vs}$, the total flux is $0.012 \mathrm{Vs}$, which is much smaller than the measured flux. This indicates that most of the current is flowing in the vacuum chamber, as described below.

A more detailed waveform for the plasma position is shown in Fig. 6(e). While the ohmic transformer current is terminated at $0.5 \mathrm{~s}$, the vertical field increases with $I_{\mathrm{PF} 26}$ and reaches the maximum at $t \sim 0.595 \mathrm{~s}$ (Fig. 6 (f)). The plasma current becomes maximum at almost the same time. The loop voltage measurements (Fig. 6(g)) suggest that the vertical field increment creates this loop voltage and induces the plasma current after ohmic coil current termination. A loop voltage of less than $1.2 \mathrm{~V}$ was actually applied before the plasma current peak. Figure 6(c) shows the fluxes representing the sum of the vacuum chamber and plasma currents, which are obtained from the measured fluxes at the inner and outer positions (Flux00 and Flux04, respectively, in Fig. 5 (f)). Here the fluxes from the 

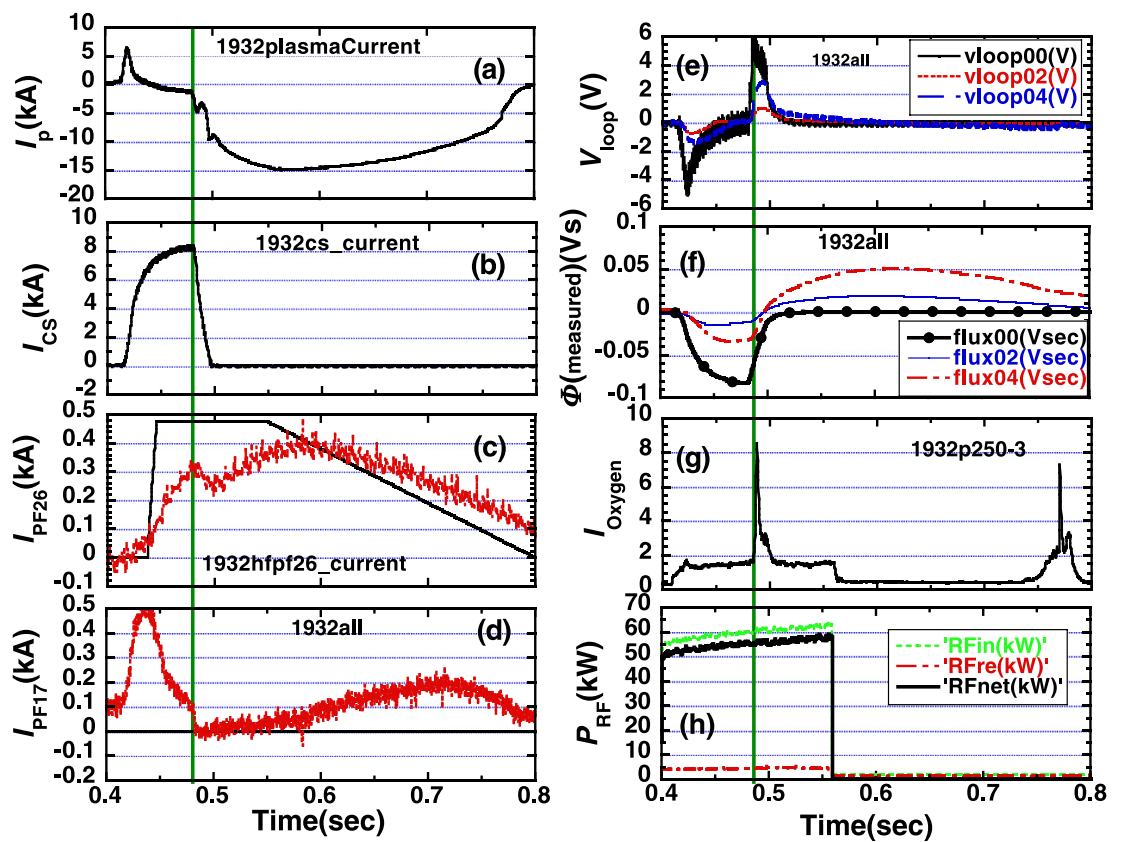

Fig. 5 Plasma current evolution in QUEST at $B_{\mathrm{t}}=0.14 \mathrm{~T}$ with one-turn cancellation coil. (a) Plasma current, (b) CS current, (c) PF26 vertical shaping coil current and set value, (d) PF17 vertical field coil current, (e) loop voltage measured at three locations, (f) flux measured at three locations, (g) oxygen impurity line, and (h) $8.2 \mathrm{GHz}$ RF power. (\#1932)

ohmic transformer and the PF26 and PF17 coils are subtracted from the measured flux ( $\Phi($ measured)) upon consideration of the coil current polarity, and then the final flux $\left(\Phi\left(I_{\mathrm{p}}+I_{\mathrm{VC}}\right)\right.$ in Fig. $\left.6(\mathrm{c})\right)$, which is the sum of the fluxes from the vacuum chamber current $I_{\mathrm{VC}}$ induced by all the poloidal coils and the plasma current $I_{\mathrm{p}}$, is given by

$$
\left\{\begin{aligned}
\Phi_{\mathrm{IU}}\left(I_{\mathrm{p}}+I_{\mathrm{VC}}\right)= & \Phi_{\mathrm{IU}}(\text { measured })-\Phi_{\mathrm{IU}-\mathrm{CS}} \\
& -\Phi_{\mathrm{IU}-\mathrm{PF} 26}-\Phi_{\mathrm{IU}-\mathrm{PF} 17} \\
\Phi_{\mathrm{OU}}\left(I_{\mathrm{p}}+I_{\mathrm{VC}}\right)= & \Phi_{\mathrm{OU}}(\text { measured })-\Phi_{\mathrm{OU}-\mathrm{CS}} \\
& -\Phi_{\mathrm{OU}-\mathrm{PF} 26}-\Phi_{\mathrm{OU}-\mathrm{PF} 17}
\end{aligned}\right.
$$

where the fluxes between the two flux loops and the PF coils are given by

$$
\begin{aligned}
& \Phi_{\mathrm{IU}-\mathrm{CS}}=-10.09 \times 10^{-3} I_{\mathrm{CS}}(\mathrm{kA}), \\
& \Phi_{\mathrm{IU}-\mathrm{PF} 26}=+4.4127 \times 10^{-3} I_{\mathrm{PF} 26}(\mathrm{kA}), \\
& \Phi_{\mathrm{IU}-\mathrm{PF} 17}=+9.345 \times 10^{-3} I_{\mathrm{PF} 17}(\mathrm{kA}), \\
& \Phi_{\mathrm{OU}-\mathrm{CS}}=-10.05 \times 10^{-3} I_{\mathrm{CS}}(\mathrm{kA}), \\
& \Phi_{\mathrm{OU}-\mathrm{PF} 26}=+148.55 \times 10^{-3} I_{\mathrm{PF} 26}(\mathrm{kA}), \text { and } \\
& \Phi_{\mathrm{OU}-\mathrm{PF} 17}=+54.98 \times 10^{-3} I_{\mathrm{PF} 17}(\mathrm{kA}),
\end{aligned}
$$

Here, the subscripts "IU" and "OU" indicate the inner upper and outer upper flux loops, respectively. Because the estimated values in Fig. 6(c) are based on both measured and calculated values for the PF coil currents, they are sensitive to coil position errors.

The inner flux (INFLUXVP) and the outer flux (OUTFLUXVP) cross zero when the ohmic coil bias current saturates, as shown in Fig. 6 (c). Plasma breakdown was expected to occur in this phase because of the negligible vacuum chamber current effect. In the plasma current ramp-up phase, because of the ramp-down of the ohmic coil current, the vacuum chamber currents flow in the same direction as the plasma current. After the ohmic coil current is reduced to zero, the vacuum chamber current still flows and is eventually damped away later.

Furthermore, the delay time effects due to the vacuum chamber on the flux loop from the ohmic, PF26, and PF17 coil currents have been taken into account in the plasma position measurements. For example, two time constants of $1.17 \mathrm{~s}$ and $8.49 \mathrm{~s}$ were obtained for the inner upper flux (Flux00) in separate discharges without the plasma current, and values of $9.46 \mathrm{~s}$ and $13.5 \mathrm{~s}$ were obtained for the outer upper flux (Flux04); these values were used for conversion from the ohmic coil current. The fluxes $\Phi_{\mathrm{OU}-\mathrm{CS}-\mathrm{VC}}$, $\Phi_{\mathrm{OU}-\mathrm{PF} 26-\mathrm{VC}}$, and $\Phi_{\mathrm{OU}-\mathrm{PF} 17-\mathrm{VC}}$, with the vacuum chamber effect obtained in this way, were subtracted from the measured values, yielding the flux from the plasma current alone, as shown in the following relationships.

$$
\left\{\begin{aligned}
\Phi_{\mathrm{IU}}\left(I_{\mathrm{p}}\right)= & \Phi_{\mathrm{IU}}(\text { measured })-\Phi_{\mathrm{IU}-\mathrm{CS}-\mathrm{VC}} \\
& -\Phi_{\mathrm{IU}-\mathrm{PF} 26-\mathrm{VC}}-\Phi_{\mathrm{IU}-\mathrm{PF} 17-\mathrm{VC}} \\
\Phi_{\mathrm{OU}}\left(I_{\mathrm{p}}\right)= & \Phi_{\mathrm{OU}}(\text { measured })-\Phi_{\mathrm{OU}-\mathrm{CS}-\mathrm{VC}} \\
& -\Phi_{\mathrm{OU}-\mathrm{PF} 26-\mathrm{VC}}-\Phi_{\mathrm{OU}-\mathrm{PF} 17-\mathrm{VC}}
\end{aligned}\right.
$$

Here, the subscript "VC" indicates the vacuum chamber effect. Figure 6(d) shows the fluxes from the plasma current alone. The signal before plasma breakdown is almost canceled out. Note that because the estimated values in Fig. 6 (d) are based on all the measured values, they are not affected by coil position errors.

In Fig. 6 (e), the plasma position was derived from these two fluxes in Fig. 6 (d). Up-down symmetry was as- 


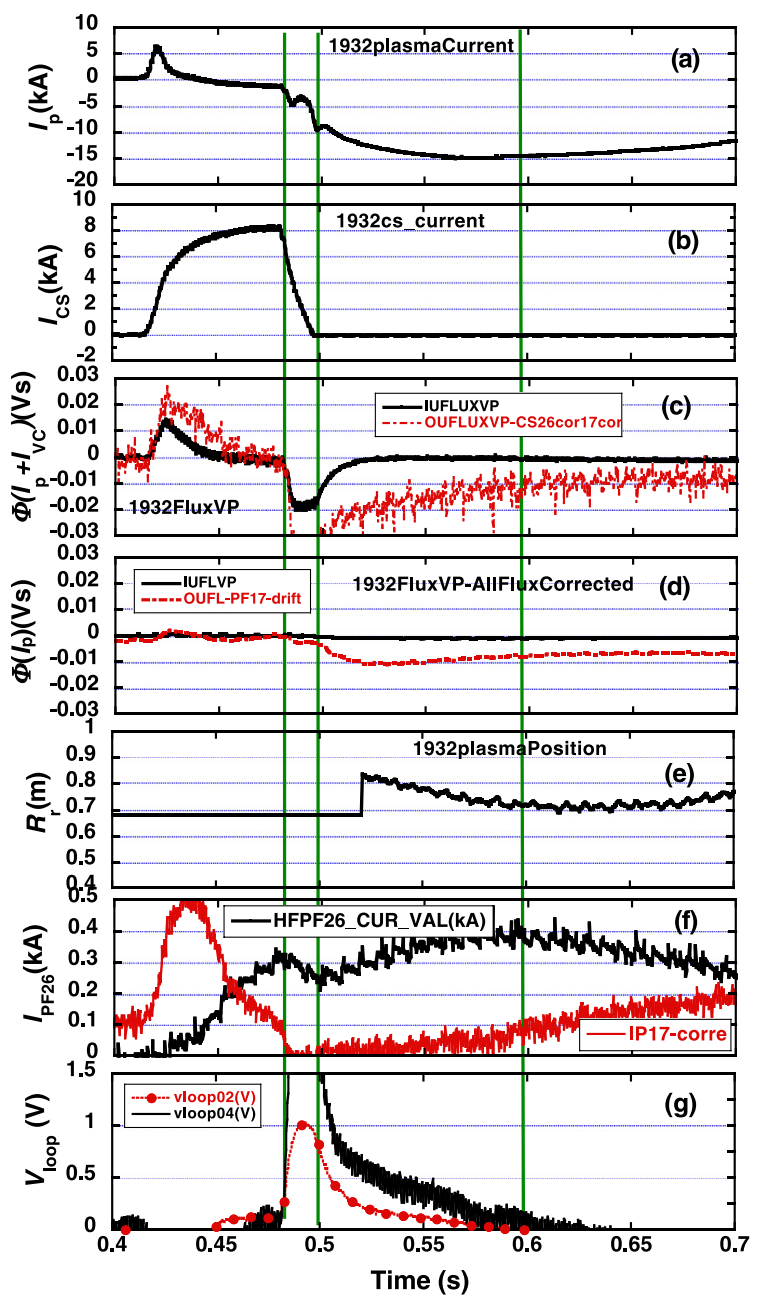

Fig. 6 Detailed versions of waveforms in Fig. 5. (a) Plasma current, (b) CS current, (c) flux consisting of vacuum chamber current and plasma current [red: outer flux (Flux04); black: inner flux (Flux00)], (d) flux due to plasma current alone [red: outer flux (Flux04); black: inner flux (Flux00)], (e) plasma position, (f) PF26 and PF17 coil currents, and $(\mathrm{g})$ loop voltage measured at Flux02 and 04 positions. Third vertical line marks zero loop voltage. (\#1932)

sumed because the up and down fluxes do not differ, and the fast camera shows no upward shift in the plasma current. Using the least square method, for the fluxes obtained in Fig. 6 (d), and the single current loop approximation, the plasma position was calculated as shown in Fig. 6(e). Because the plasma position before the vacuum chamber current decay ( $t<0.52 \mathrm{~s}$ ) may not be accurate, that phase is omitted in this analysis. The plasma position is approximately around $R \sim 0.72 \mathrm{~m}$ at $t \sim 0.6 \mathrm{~s}$ and gradually shifts inward. For this parameter, the inner plasma's minor radius is a $\sim 0.50 \mathrm{~m}$ because the plasma is limited by an inner limiter placed at $0.22 \mathrm{~m}$. An almost circular plasma is formed with an aspect ratio of $A \sim 0.72 / 0.50 \sim 1.44$, and the edge safety factor could be estimated as $q \sim 5 a^{2} B_{\mathrm{t}} / R I_{\mathrm{p}} \sim 16$. Note that because the plasma edge cannot be seen by the
TV camera during the full plasma current phase owing to the low density, a cross check of the plasma position awaits higher-density operation. Although we used only two flux loops, several flux loops are expected to provide a plasma boundary in the near future by using the Cauchy condition surface (CCS) method [26]. Therefore, the above estimation of the plasma position is only tentative and is not yet accurate.

As the circuit parameters of the shaping and vertical field coils (PF26 and PF17, respectively) have been set for long-duration steady-state operation in this first experimental campaign, a quick response to match the plasma current ramp-up in $20 \mathrm{~ms}$ is not possible, leading to the early application of the vertical field coil current. In addition, because the coupling between the ohmic coil and the PF26 (Fig. 5 (c)) and PF17 (Fig. 5 (d)) coils are quite strong, these coil currents are induced unintentionally because of the fact that the switches in these circuits are already on owing to the present circuit limitation. Further optimization of these circuit parameters would increase the plasma current.

\subsection{Smaller ohmic coil bias current of $I_{\mathrm{CS}}=$ $5 \mathbf{k A}$}

To decrease the coil coupling and optimize the ohmic transformer current, the ohmic coil bias current was reduced to $I_{\mathrm{CS}}=5 \mathrm{kA}$; the results are shown in Fig. 7. The RF application time was delayed and was shorter than that in Fig. 5. Therefore, the initial negative plasma current was not induced. The resulting plasma current evolution is smoother, and a slightly larger and longer plasma current has been obtained by applying a longer and larger vertical field. This is the best discharge waveform in the first ohmic discharge experimental campaign. As the ohmic coil bias current $I_{\mathrm{CS}}$ is reduced, the coupling between the PF26 and PF17 coils decreases, as seen in Fig. 7 (d). When the bias current $I_{\mathrm{CS}}$ is further reduced to $4 \mathrm{kA}$, the coupling between the PF26 and ohmic transformer coils decreases, and a plasma current is successfully established.

Figure 8 shows more detailed waveforms. Because the loop voltage is also positive until the plasma current peak, it is clearly understood that the vertical field drives the plasma current for a long time after the ohmic coil current is switched off, which was also done previously. The fluxes from the plasma and vacuum chamber currents and from the plasma current alone are shown in Figs. 8 (c) and $8(\mathrm{~d})$, respectively. The plasma position remains stable at around $0.7 \mathrm{~m}$ for a long time in the outward position with a better $n$-index area. A plasma position that remains in the stable regime with a positive $n$-index, defined as $n=-R / B_{\mathrm{v}}\left(\mathrm{d} B_{\mathrm{v}} / \mathrm{d} R\right)$, may yield a longer plasma current without feedback control.

A fast TV camera monitored the plasma cross section and position only in the early phase starting $3 \mathrm{~ms}$ after plasma current start-up, as shown in Fig. 9. The plasma 

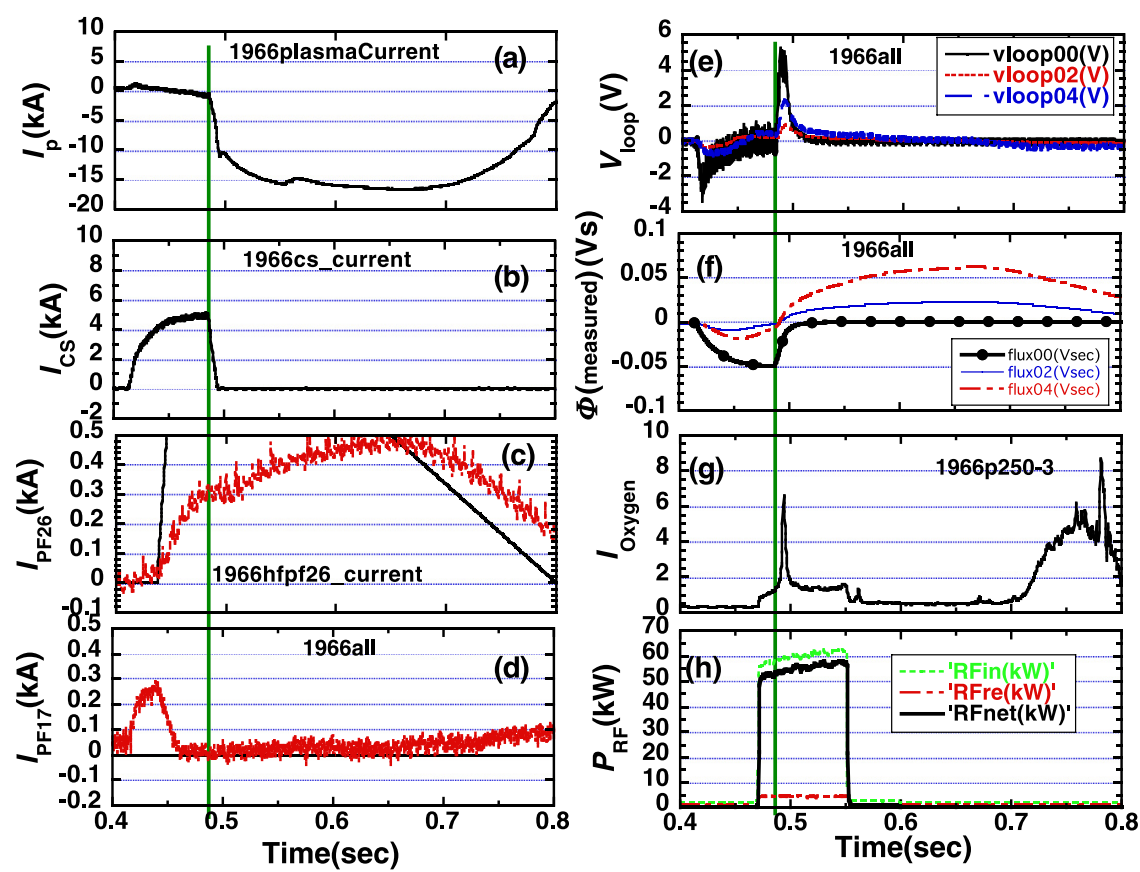

Fig. 7 Plasma current evolution in QUEST at $B_{\mathrm{t}}=0.14 \mathrm{~T}$ with smaller ohmic transformer bias current. (a) Plasma current, (b) CS current, (c) PF26 vertical shaping coil current and set value, (d) PF17 vertical field coil current, (e) loop voltage measured at three locations, (f) flux measured at three locations, (g) oxygen impurity line, and (h) $8.2 \mathrm{GHz}$ RF power. (\#1966)

current seems to start on the central ohmic transformer of $R \sim 0.23 \mathrm{~m}$ at $t=0.4912 \mathrm{~s}$ and expands outward to $R \sim 0.44 \mathrm{~m}$ at $t=0.4927 \mathrm{~s}$; a closed flux surface is clearly seen at $0.4942 \mathrm{~s}$. The radius of the central toroidal limiter $(0.22 \mathrm{~m})$ suggests that the center of the plasma's cross section is at around $R=0.55 \sim 0.6 \mathrm{~m}$ at $t=0.4942 \mathrm{~s}$. After this, the plasma boundary is unclear; for example, at $t=0.55 \mathrm{~s}$ (Fig. 9(d)), the plasma boundary is not visible because of the low-density operation. Because the plasma position in the later phase is at around $R \sim 0.70 \mathrm{~m}$, as measured by the two flux loops, the plasma initially moves outward and then remains at a stable position. Because the positions obtained by these two methods do not overlap, an accurate comparison cannot be made in this situation.

Although density measurements were performed using reflectometry, it was difficult to determine the density because of the small number of fringes. In the near future, a 2-mm interferometer will be available to measure the density. However, hard X-ray measurements of up to $100 \mathrm{keV}$ using a CdZnTe semiconductor detector, as shown in Fig. 10 (a) at $t=0.5 \mathrm{~s}$, suggest that the density could be low. The detector is placed on QUEST's equatorial plane and views the forward emission from runaway electrons tangentially; the energy range is limited to $100 \mathrm{keV}$ in this experiment. Runaway electrons with various energies were created after ohmic coil current decay, as shown in Fig. 10 (b), where the time resolution is as large as $0.1 \mathrm{~s}$. This may be due to acceleration by the vertical field induction effect. The times required to reach the peak values of the plasma current and hard X-rays are almost the same,
$0.65 \mathrm{~s}$.

When the vertical field drives the plasma current, additional heating power is necessary to prevent the plasma current from shifting inward [2]. However, in this QUEST experiment, no additional heating power was applied during the vertical field current ramp-up phase. The electric field, created during plasma current ramp-up by the vertical field, accelerates the electrons to higher energies, as shown in Fig. 10 (b). This increases the plasma energy, balancing the inward force of the vertical field. Thus, this new mechanism for ramping up the plasma current without additional heating power may work only in the low-density regime.

\section{Discussions and Summary}

The operational waveform achieved here is suitable for the future steady-state operation in QUEST because the ohmic transformer is already switched off. If the density is below the cut-off value, electron cyclotron current drive (ECCD) can sustain the plasma current. If the density is higher than the cut-off value, EBCD would drive the plasma current by using the new EBCD antenna configuration [27], as shown schematically in the operation scenario in Fig. 11.

In the present discharges, no plasma breakdown occurs without RF power. Thus, we see that RF power contributes to the breakdown and the plasma current formation is quite reproducible. At the resonant position of $0.33 \mathrm{~m}$ in this case, the loop voltage is $V_{\mathrm{L}} \sim 4 \mathrm{~V}$, the electric field is 


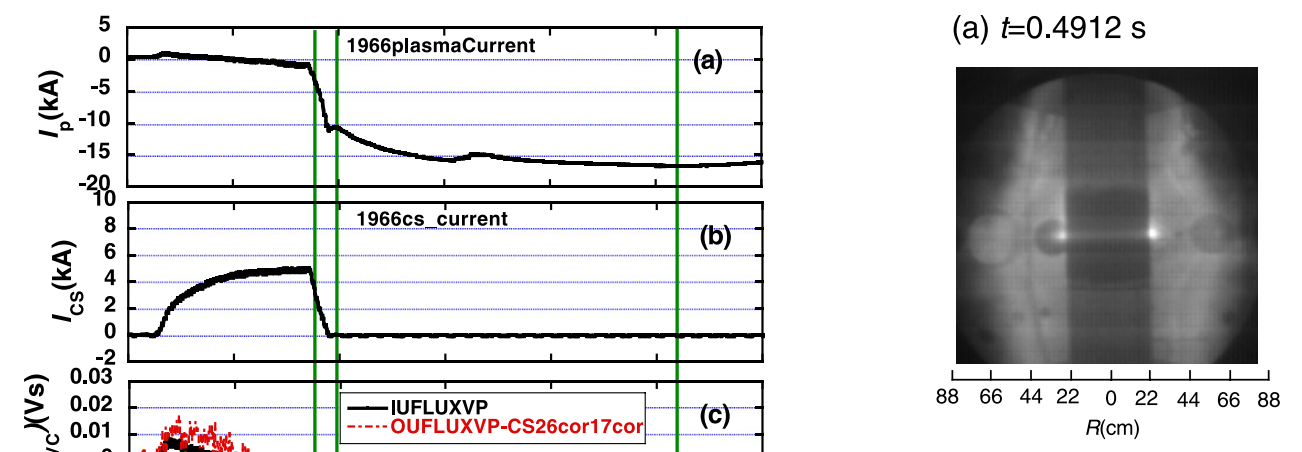

(c) $t=0.4942 \mathrm{~s}$

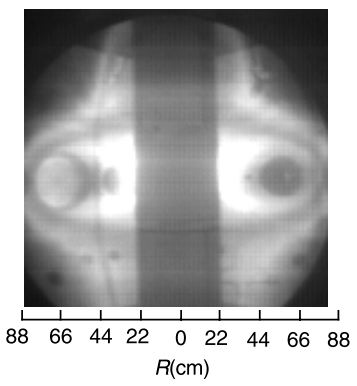

(b) $t=0.4927 \mathrm{~s}$

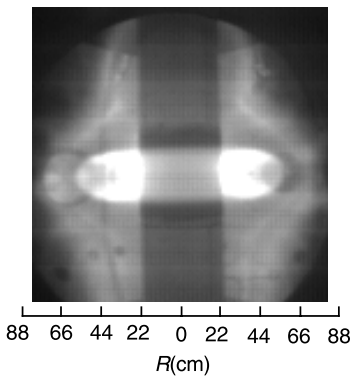

(d) $t=0.55 \mathrm{~s}$

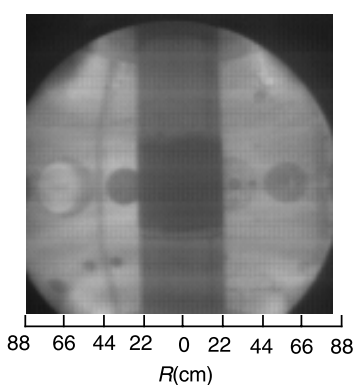

Fig. 9 Fast TV camera images in the initial plasma current startup phase. (\#1966)

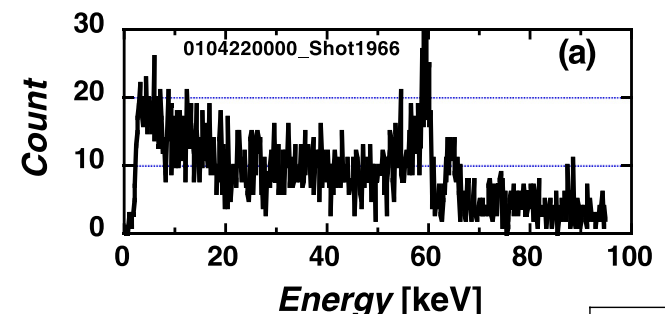

Fig. 8 Detailed versions of waveforms in Fig. 7. (a) Plasma current, (b) CS current, (c) flux consisting of vacuum chamber current and plasma current [red: outer flux (Flux04); black: inner flux (Flux00)], (d) flux from plasma current alone [red: outer flux (Flux04); black: inner flux (Flux00)], (e) plasma position, (f) PF26 and PF17 coil currents, and (g) loop voltage measured at Flux 02 and 04 positions. Third vertical line marks zero loop voltage. (\#1966).

$E=V_{\mathrm{L}} / 2 \pi R \sim 1.9 \mathrm{~V} / \mathrm{m}$, and the toroidal field is $0.28 \mathrm{~T}$; the sum of the applied vertical field and ohmic stray field is $\sim 122 \mathrm{G}$. At the resonant position, the vertical field calculated with a PF26 coil current of $I_{\mathrm{PF} 26}=0.25 \mathrm{kA}$ is $82 \mathrm{G}$ and the measured vertical field is $\sim 40 \mathrm{G}$ at $I_{\mathrm{CS}}=8 \mathrm{kA}$, as shown in Fig. 12 (d), which was obtained during the machine maintenance phase [28]. Thus, we have

$$
\frac{E B_{\mathrm{t}}}{B_{\mathrm{V}}} \sim \frac{1.942 \times 0.28}{0.0122}=44.5[\mathrm{~V} / \mathrm{m}]
$$

which is smaller than the value of $E B_{\mathrm{t}} / B_{\mathrm{v}}=76 \mathrm{~V} / \mathrm{m}$ obtained in the strong-stray field experiments in DIII-D with $3 \mathrm{~V}, 75 \mathrm{G}$, and $B_{\mathrm{t}}=2 \mathrm{~T}$ [29]. As shown in Fig. $12(\mathrm{~d})$, the measured and calculated vertical fields from the ohmic

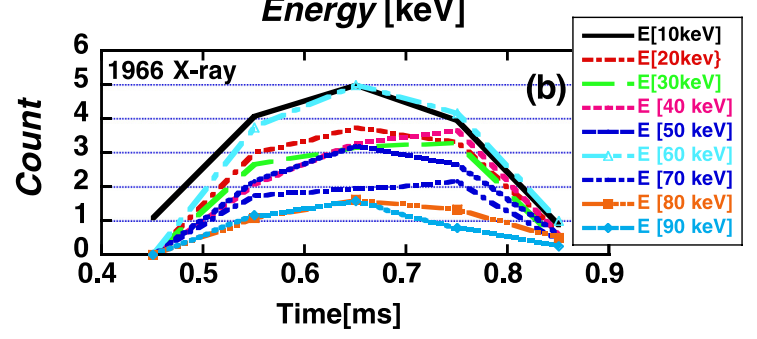

Fig. 10 Temporal evolution of hard X-rays measured by CdZnTe semiconductor detector and pulse height system. (a) Energy spectrum at $t=0.5 \mathrm{~s}$ and (b) temporal evolution at various energies. (Time resolution is $0.1 \mathrm{~s}$ for integration.) (\#1966)

coil alone are almost the same. Therefore, the vertical field from the vacuum chamber current is negligible because the total vacuum chamber current crosses zero at the breakdown phase, as shown in Figs. 6(c) and 8 (c). However, the local vacuum chamber current is not zero because of the difference in inductance between the outer and inner vacuum chambers, as described previously. Numerical calculations of the vacuum chamber current by using the computer code EDDYCAL (JAEA) have confirmed that 


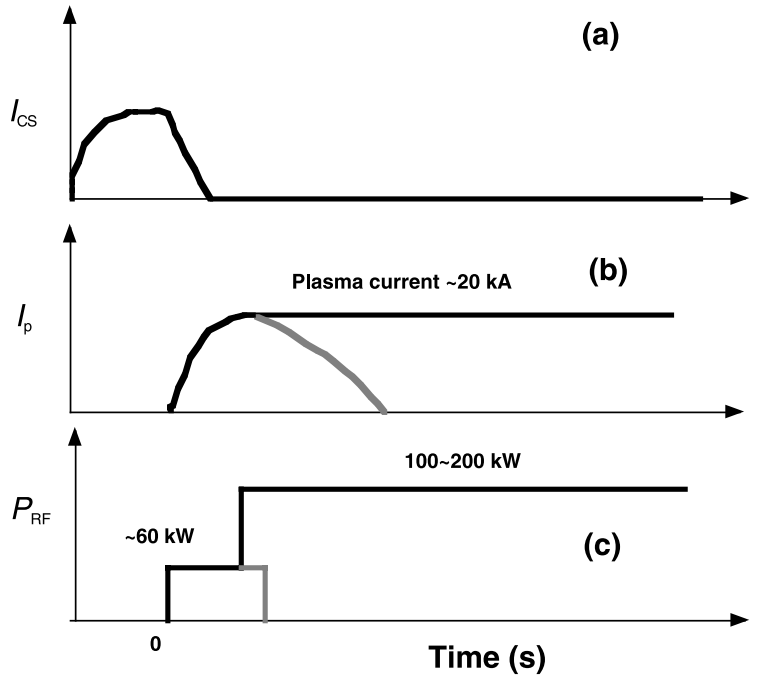

Fig. 11 Operation scenario for steady-state EBCD operation.
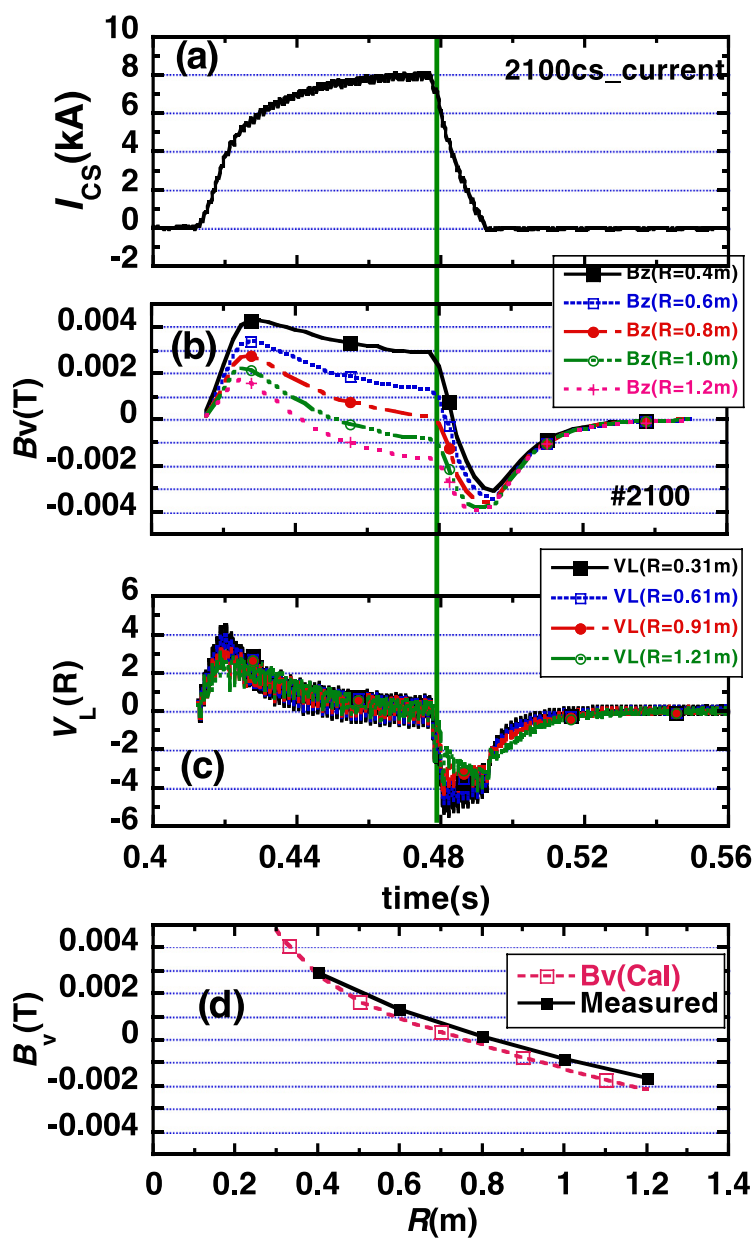

Fig. 12 Measured vertical field inside vacuum chamber for $I_{\mathrm{CS}}=$ $8 \mathrm{kA}$. (a) Ohmic coil current, (b) measured magnetic fields $B_{\mathrm{v}}$ at various positions, (c) loop voltages (\#2100) [29], and (d) measured and calculated $B_{\mathrm{v}}$ profiles at $I_{\mathrm{CS}}=8 \mathrm{kA}$.
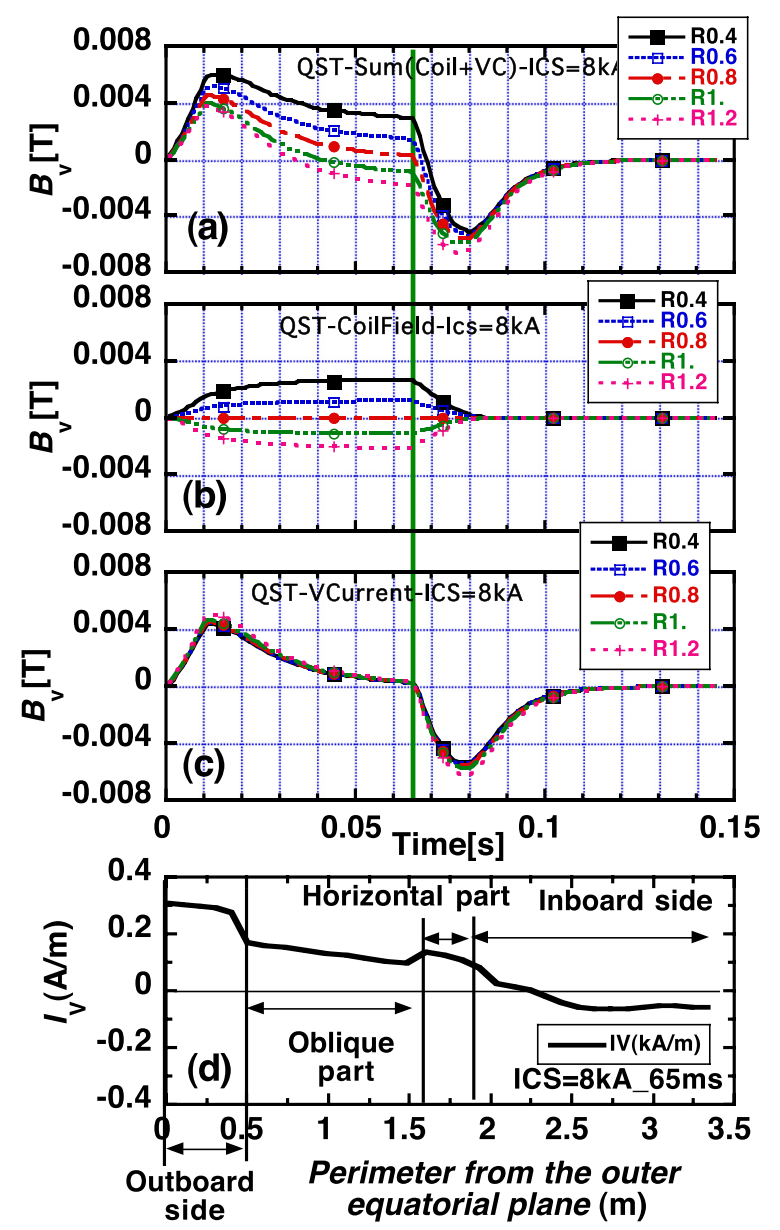

Fig. 13 Calculated vertical field inside vacuum chamber for $I_{\mathrm{CS}}=$ $8 \mathrm{kA}$. (a) Total magnetic field, (b) magnetic field from ohmic coil current without the effect of the vacuum chamber, (c) magnetic field produced by vacuum chamber current alone [29], and (d) vacuum chamber current densities in various chamber positions on upper vacuum chamber at $65 \mathrm{~ms}$.

while the vertical field from the vacuum chamber current alone crosses zero at the breakdown phase, as shown in Fig. 13 (c), the local vacuum chamber current is not zero. At $65 \mathrm{~ms}$, as shown in Fig. 13 (d), the inner vacuum chamber current reverses its polarity earlier but the outer vacuum chamber current keeps flowing in the same direction.

Because the reliable breakdown criterion is $E B_{\mathrm{t}} / B_{\mathrm{v}} \sim$ $1000 \mathrm{~V} / \mathrm{m}$ in a stray vertical field environment $[28,30]$, the obtained value is quite low. The connection length is quite short, $L \sim a_{\text {eff }} B_{\mathrm{t}} / B_{\mathrm{v}}=(0.33-0.22) \times 0.28 / 0.0122=2.5 \mathrm{~m}$, corresponding to 1.2 turns at the resonant position in the toroidal direction. Here, $a_{\mathrm{eff}}$ is defined as the distance from the resonant position to the inside limiter.

The runaway electrons created by the vertical field may lead to a high-energy electron tail distribution function, which is also favorable for EBCD. Heating power is not applied during the "ohmic clamp" phase, but the plasma current is ramped up. This may be due to the accel- 
eration of the runaway electrons by vertical field induction. However, further experiments and theoretical calculations are needed to explain this result.

The PF26 coil current waveforms during the plasma current ramp-up phase in shots \#1932 and \#1966 are different, as seen in Figs. 5 (c) and 7 (c). Because the PF26 coil current decreases from 0.3 to $0.25 \mathrm{kA}$ owing to the strong coil coupling at $I_{\mathrm{CS}}=8 \mathrm{kA}$, the plasma current has bumps. This is because both cases have similar plasma currents; however, the equilibrium field is smaller in \#1932 and so the plasma current ramp-up is not smooth. Therefore, it is important to reduce the coil coupling for better plasma control.

We achieved a plasma current of only $17 \mathrm{kA}$, which is lower than the target value. A slower ohmic current decay would increase the plasma current. Smaller inductance in the vertical field coil with a half turn number would allow easier plasma position equilibration than that in the present discharge during plasma current ramp-up, which also leads to a larger plasma current. Feedback control, in turn, plays an important role in achieving a larger plasma current, longer pulse, and steady-state operation, and is under development using the CCS method [26].

We have described the initial ohmic discharge experiments in QUEST assisted with RF heating power. We demonstrated that the $\mathrm{CC}$ was very efficient and reliable in generating a plasma current in the early phase of the ohmic discharge experiments, as expected, although the null field regime was not created because a vertical field was applied in the breakdown phase. Therefore, the RF power strongly assisted this ohmic discharge start-up. The initial discharge waveform is very suitable for achieving the future steady-state operation by ECCD or EBCD because the ohmic coil current is already switched off and is zero in the early phase, in which the vertical field drives the plasma current during the zero phase of the ohmic transformer current and facilitates the transition from the ohmic phase to the steady-state phase. This "ohmic clamp" experiment demonstrates that a vertical field without any heating power can ramp up the plasma current. Genuine CS-less operation using vertical field coils is also planned for the future steady-state operation, which is crucial in the future ST operation.

This work was performed with the support and under the auspices of the NIFS bi-directional collaborative research program (NIFS07KUTR020).

[1] Y-K M. Peng et al., Fusion Technol. 30, 1372 (1996).

[2] O. Mitarai, Plasma Phys. Control. Fusion 41, 1469 (1999).
[3] O. Mitarai and Y. Takase, Fusion Sci. Technol. 43, 67 (2003).

[4] O. Mitarai, R. Yoshino and K. Ushigusa, Nucl. Fusion 10, 1257 (2002).

[5] Y. Takase et al., J. Plasma Fusion Res. 78, 717 (2002).

[6] S. Shiraiwa et al., Phys. Rev. Lett. 92, 035001 (2004).

[7] O. Mitarai, Y. Takase, A. Ejiri, S. Shiraiwa et al., J. Plasma Fusion Res. 80, 549 (2004).

[8] A. Sykes et al., in 19th IEEE/NPSS Symposium on Fusion Engineering (SOFE) (Atlantic City, USA, January 22-25), (2002) p.125.

[9] M. Ushigome, S. Ide, S. Itoh, E. Jotaki, O. Mitarai, S. Shiraiwa, T. Suzuki, Y. Takase et al., Nucl. Fusion 46, 207 (2006).

[10] O. Mitarai, C. Kessel and A. Hirose, JEEE 129, No.9, Sec. A, 605 (2009).

[11] K. Hanada et al., in 22nd IAEA Fusion Energy Conference, (13-8, Oct. 2008, Geneve, Switzerland) FT/P3-25.

[12] C. Neumeyer et al., Fusion Eng. Des. 54, 275 (2001).

[13] J.J. Ramos, MIT Plasma Fusion Center report PFC/RR-889 (1988).

[14] B.J. Xiao, D.A. Humphreys et al., Fusion Eng. Des. 83, 181 (2008).

[15] J.A. Leuer, N.W. Eidiettis et al., General Atomic report GA-A26492 (July 2009); Yeong-Kook Oh et al., Fusion Eng. Des. 83, 181 (2008).

[16] Y. Gribov et al., in chapter 8: Plasma Operation and Control, Nucl. Fusion 47, S385 (2007).

[17] M. Matsukawa et al., in Asia Plasma and Fusion Association (APFA) Conference (Oct 27-29. 2009, Aomori, Japan) P27p1-17.

[18] W. Køppendørfer et al., Nucl. Eng. Des./Fusion 3, 265 (1986).

[19] S. Kinoshita et al., General Atomic report GA-A19584, (Dec. 1989).

[20] R. Yoshino et al., Fusion Eng. Des. 24, 375 (1994).

[21] K. Nakamura, Private communications (2010 March 25) because of no official documentation.

[22] S. Shiraiwa, "A study of electron Bernstein wave heating for diagnostics and heating of spherical tokamak plasmas", PhD theses (Univ. of Tokyo, April 27, 2007).

[23] V.K. Gusev et al., Nucl. Fusion 41, 919 (2001).

[24] Y.C. Saxena and SST-1 Team, Nucl. Fusion 40, 1069 (2000).

[25] V.A. Korotkov et al., Fusion Eng. Des. 56-57, 831 (2001).

[26] K. Kurihara, Fusion Eng. Des. 51-52, 1049 (2000).

[27] H. Idei et al., "Ray tracing and Fokker-Plank analyses for electron Bernstein wave heating and current drive in QUEST", ICCP 2009 (Fukuoka).

[28] O. Mitarai, K. Nakamura et al., J. Plasma Fusion Res. SERIES 9, 100 (2010); in Asia Plasma and Fusion Association (APFA) Conference (Oct 27-29. 2009, Aomori, Japan) P27p2-01.

[29] B. LLoyd et al., Nucl. Fusion 31, 2031 (1991).

[30] V. Erkman and U. Gaspanrino, Plasma Phys. Control. Fusion 36, 1869 (1994). 